\title{
Analytical solutions for Navier-Stokes equations in the cylindrical coordinates
}

\author{
V. Adanhounme ${ }^{\mathrm{a} 1}, \mathrm{~A}$. Adomou ${ }^{\mathrm{b}}$ and F. P. Codo $^{\mathrm{c}}$ \\ ${ }^{a}$ International Chair of Mathematical Physics and Applications, \\ (ICMPA-UNESCO Chair), Université d'Abomey-Calavi, 072 B.P. 50 Cotonou, Republic of Benin \\ E-mail: adanhounm@yahoo.fr \\ b Institut Universitaire de Technologie \\ Université d'Abomey-Calavi, B.P.133 Lokossa, Republic of Benin \\ E-mail: denisadomou@yahoo.fr \\ 'Laboratoire d'Energétique et de Mécanique Appliquée, \\ (EPAC), Université d'Abomey-Calavi, 01 B.P.2009 Cotonou, Republic of Benin \\ E-mail: fdepaule2003@yahoo.fr
}

Keywords: Navier-Stokes equations; No-slip boundary conditions; Convection.

\begin{abstract}
We consider the problem of convective heat transport in the incompressible fluid flow and the motion of the fluid in the cylinder which is described by the Navier-Stokes equations with the heat equation.The exact solutions of the Navier-Stokes equations, the temperature field and the vorticity vector are obtained.
\end{abstract}

\section{INTRODUCTION}

The fluid mechanics studies the behavior at every point within the domain under various physical conditions. For describing the physical phenomena in fluid mechanics one uses the mathematical model of motion such as Navier-Stokes equations. It is a well known fact that a few exact solutions of the Navier-Stokes equations are known even now. This has been largely due to the complexity of the system of differential equations. In the absence of a general solution it is often convenient to experiment with models to obtain information on the flow phenomena e.g. the velocity distribution, flow pattern, pressure losses, etc. In [1] one applied the "background" method to the arbitrary Prandtl number problem to derive a scaling lower bound on the space-time averaged temperature of the layer along with an explicit prefactor and one used a multiple boundary layer asymptotic theory to sharpen the estimate, increasing the prefactor in the lower bound by a factor. More recently, the existence of the global regular solutions to Navier-Stokes equations is proven in [8]. In this paper we investigate the analytical solutions to Navier-Stokes equations in cylindrical coordinates since the problem of transport of mass,momentum and heat in the case of flow is of great importance for engineering applications. The rest of this paper is organized as follows :in the next section we present the details of the models we analyze.In the section 3 we present the solutions to the NavierStokes equations and to the heat equation .In the conclusion, we summarize our results.

\section{Governing equations}

The fluid layer is confined between two parallel plates of horizontal extent $L_{x}$ and $L_{y}$ separated by vertical $(z)$ distance $(d)$. The no-slip upper and lower plates are held at fixed temperatures $T_{0}$ and $T_{1}$ respectively A uniform volumetric heat flux $H$ (with units power/volume) is pumped into the layer. The governing equations for the velocity field $u$, the pressure $p$ and the temperature $T$ in the standard Boussinesq approximation are[1]:

$$
\begin{array}{ll}
\frac{\partial v}{\partial t}+v \cdot \Delta v=-\nabla p+v \nabla^{2} v+\hat{k} g \alpha T & \text { in } \Omega^{\tau} \equiv \Omega \times(0, \tau) \\
\frac{\partial T}{\partial t}+v \cdot \nabla T=\kappa \nabla^{2} \mathrm{~T}+\gamma & \text { in } \Omega^{\tau}
\end{array}
$$


$\nabla \cdot v=0$

in $\Omega^{\tau}$

with the boundary conditions

$v=0$ on $S^{\tau} \equiv S \times(0, \tau)$

$\left.T\right|_{z=0}=T_{0} ;\left.\quad T\right|_{z=d}=T_{1}$

where $\quad v=v(x, t)=\left(v_{1}(x, t), v_{2}(x, t), v_{3}(x, t)\right) \in R^{3} \quad$ is the velocity of the fluid; $p=p(x, t) \in R$ the pressure; $T=T(x, t) \in R$ the temperature; $\hat{k}=(0,0,1) ; v$ is the viscosity, $g$ is the acceleration of gravity along the $z$ axis (in the $\hat{k}$-direction ), $\alpha$ is the thermal expansion coefficient, $\mathrm{k}$ is the thermal diffusion coefficient and $\gamma=\frac{H}{\rho c}$, where $\rho$ is the density and $c$ is the specific heat capacity of the fluid. We impose periodic boundary conditions in the horizontal directions with periods $L_{x}$ and $L_{y}$. Using $\frac{d^{2}}{\kappa}$ as the unit of time, $d$ as the unit of length and $\frac{\gamma d^{2}}{\kappa}$ as the unit of temperature, the governing equations are put into the nondimensional form:

$\frac{1}{p r}\left(\frac{\partial v}{\partial t}+v \cdot \nabla v\right)+\nabla p=\nabla^{2} v+R a T \hat{k}$

$\frac{\partial T}{\partial t}+v \cdot \nabla T=\nabla^{2} \mathrm{~T}+1$

where $\operatorname{Pr}=\frac{v}{\kappa}$ is the Prandtl number and $R a=\frac{g \alpha d^{5}}{\kappa^{2} v}$ is the heat Rayleigh number. By the dot we denote the scalar product in $R^{3}$ In order to describe the domain $\Omega$ and the motion of the fluid, we introduce the cylindrical coordinates $g, \varphi, z$ which are associated with the cartesian coordinates $x_{1}$, $x_{2}, x_{3}$ by the relations:

$x_{1}=g \cos \varphi ; x_{2}=g \sin \varphi ; x_{1}=z ; g$ denotes the distance from the distinguished axis expressed in the cylindrical coordinates $g, \varphi, z$ as the $z$-axis of symmetry/

Then, we adopt the following assumptions :

- the domain $\Omega$ is defined as:

$$
\begin{aligned}
& \Omega=\left\{x \in R^{3}: \varrho<R ; 0<z<d ; \varphi \in[0,2 \pi] ; R=\text { const }\right\} ; \\
& S=S_{1} \cup S_{2} \quad \text { where } \\
& S_{1}=\left\{x \in R^{3}: \varrho=R ; \quad 0<z<d ; \varphi \in[0,2 \pi]\right\} ; \\
& S_{2}=\left\{x \in R^{3}: \varrho<R, z=0, z=d, \varphi \in[0,2 \pi]\right\} .
\end{aligned}
$$

- the cylindrical components of any vector-valued function $v$ are defined by:

$$
\begin{aligned}
& v_{r}=v \cdot e_{r} ; v_{\varphi}=v \cdot e_{\varphi} ; v_{z}=v \cdot e_{z}, \text { where } \\
& e_{r}=(\cos \varphi, \sin \varphi, 0) ; e_{\varphi}=(-\sin \varphi, \cos \varphi, 0) ; e_{z}=(0,0,1) ; r=\frac{e}{R}
\end{aligned}
$$

- the incompressible viscous fluid flows are axisymmetric and helical .

Then the boundary conditions in the nondimensional form become :

$$
v=0
$$

$$
\text { on } \hat{S}^{\tau} \equiv{\widehat{S_{1}}}^{\tau} \cup{\widehat{S_{2}}}^{\tau} \text { where }
$$


$\widehat{S}_{1}^{\tau}=\widehat{S}_{1} \times(0, \tau) ; \widehat{S}_{2}^{\tau}=\hat{S}_{2} \times(0, \tau) ;$

$\widehat{S_{1}}=\left\{x \in R^{3}: r=1, \quad 0<z<1 ; \varphi \in[0,2 \pi]\right\} ;$

$\widehat{S_{2}}=\left\{x \in R^{3}: r<1: z=0 ; z=1 ; \varphi \in[0,2 \pi]\right\}$.

$\left.T\right|_{z=0}=\tilde{T} ;\left.\quad T\right|_{z=1}=0 \quad$ where $\tilde{T}=\left(\frac{\kappa}{\gamma d^{2}}\right)\left(T_{0}-T_{1}\right)$

For completeness, we write explicitly the three dimensional equations in cylindrical coordinates $(r, z)[2][3]$ :

$\frac{1}{p_{r}}\left(\frac{\partial v_{r}}{\partial t}+v_{r} \frac{\partial v_{r}}{\partial r}-\frac{v_{\varphi}^{2}}{r}+v_{z} \frac{\partial v_{r}}{\partial z}\right)=-\frac{\partial p}{\partial r}+\frac{\partial}{\partial r}\left(\frac{1}{r} \frac{\partial\left(r v_{r}\right)}{\partial r}\right)+\frac{\partial^{2} v_{r}}{\partial z^{2}} ;$

$\frac{1}{p_{r}}\left(\frac{\partial v_{\varphi}}{\partial t}+v_{r} \frac{\partial v_{\varphi}}{\partial r}+\frac{v_{r} v_{\varphi}}{r}+v_{z} \frac{\partial v_{\varphi}}{\partial z}\right)=\frac{\partial}{\partial r}\left(\frac{1}{r} \frac{\partial\left(r v_{\varphi}\right)}{\partial r}\right)+\frac{\partial^{2} v_{\varphi}}{\partial z^{2}} ;$

$\frac{1}{P_{r}}\left(\frac{\partial v_{z}}{\partial t}+v_{r} \frac{\partial v_{z}}{\partial r}+v_{z} \frac{\partial v_{z}}{\partial z}\right)=R a T-\frac{\partial p}{\partial z}+\frac{1}{r} \frac{\partial}{\partial r}\left(r \frac{\partial v_{z}}{\partial r}\right)+\frac{\partial^{2} v_{z}}{\partial z^{2}} ;$

$\frac{\partial T}{\partial t}-\frac{1}{r} \frac{\partial \psi}{\partial z} \frac{\partial T}{\partial r}+\frac{1}{r} \frac{\partial \psi}{\partial r} \frac{\partial T}{\partial z}=\frac{1}{r} \frac{\partial}{\partial r}\left(r \frac{\partial T}{\partial r}\right)+\frac{\partial^{2} T}{\partial z^{2}}+1 ;$

$\frac{1}{r} \frac{\partial}{\partial r}\left(r \frac{\partial \psi}{\partial r}\right)+\frac{\partial^{2} \psi}{\partial z^{2}}-\frac{\psi}{r^{2}}=\frac{1}{r}\left(\frac{\partial^{2} \psi}{\partial z^{2}}+\frac{\partial^{2} \psi}{\partial r^{2}}-\frac{1}{r} \frac{\partial \psi}{\partial r}\right)$,

Where $\psi$ is the stream function.

Our aim is to construct the exact solutions to these equations.

\section{Analytical solutions}

Now, we can formulate the main results of the paper.

\section{Proposition 1.}

The solution of the equation (14) satisfying the boundary conditions (8) has the following form: $\psi(r, z, t)=\frac{z \varepsilon\left(z-z^{2}\right)}{\alpha(t)(r-1) \sqrt{z^{2}+r^{2}}} \quad$ in $\widehat{\Omega}^{\tau} \cup \widehat{\mathrm{S}}_{2}^{\tau}$ $\psi(1, z, t)=0 \quad$ on $\hat{\mathrm{S}}_{1}^{\tau}$

where $\widehat{\Omega}^{\tau}=\widehat{\Omega} \times(0, \tau), \widehat{\Omega}=\left\{x \in R^{3} ; r<1,0<z<1, \varphi \in[0,2 \pi]\right\}$,

the function $\alpha: t \mapsto \alpha(t)$ is positive such that $\forall t>0, \psi(r, z, t)$ remains a finite quantity and $\varepsilon(y)$ a sign function.

\section{Proof}

As the fluid flows are axisymmetric and helical, we can write [3] :

$v(r, z, t)=\operatorname{rot}\left(\frac{\psi(r, z, t)}{r} e_{\varphi}\right)+\left(r v_{\varphi}\right) e_{\varphi}$

Applying the operator rot to the relation (17) and using the explicit relations between the cylindrical components of the vorticity and velocity, we get :

$\Delta\left(\frac{\psi(r, z, t)}{r}\right)=\frac{\partial v_{z}}{\partial r}-\frac{\partial v_{r}}{\partial z}$,

where $\Delta$ is the laplacian in cylindrical coordinates $r, z$.

Using the divergence-free condition

$$
\nabla . v(r, z, t)=0 \text { i.e. } \quad \frac{\partial\left(r v_{r}\right)}{\partial r}+\frac{\partial\left(r v_{z}\right)}{\partial z}=0
$$


And the stream function $\psi$ for the velocity through the relations

$v_{r}(r, z, t)=-\frac{1}{r} \frac{\partial \psi}{\partial z}(r, z, t), \quad v_{z}(r, z, t)=\frac{1}{r} \frac{\partial \psi}{\partial r}(r, z, t)$,

then the equation (18) is equivalent to (14) which leads to :

$\left(r^{2}-r\right) \frac{\partial^{2} \psi}{\partial r^{2}}+\left(r^{2}-r\right) \frac{\partial^{2} \psi}{\partial z^{2}}+(r+1) \frac{\partial \psi}{\partial r}-\psi=0$

Putting $\psi(r, z, t)=\frac{\alpha f(s)}{r-1}, s=\frac{\alpha z}{r}, \alpha=\alpha(t), \forall(r, z, t) \in \widehat{\Omega}^{\tau} \cup{\widehat{S_{2}}}_{2}^{\tau}$ the equation (19) leads to $\left(s^{2}+\alpha^{2}\right) f^{\prime \prime}(s)+3 s f^{\prime}(s)=0$

which produces the solution :

$\psi(r, z, t)=\frac{C_{1} z \varepsilon\left(z-z^{2}\right)}{\alpha(t)(r-1) \sqrt{z^{2}+r^{2}}}+\frac{C_{2} \alpha}{r-1}$

where $\varepsilon(\xi)$ is the sign function and $C_{1}, C_{2}=$ const.

On $\hat{S}_{1}{ }^{\tau}$ the equation (14) has the following solution :

$\psi(1, z, t)=0, \forall t \in(0, \tau), \quad 0<z<1$.

Using the boundary conditions $v_{\mid z=0,1}=0$ and $v(r, z, t)=\left(v_{r}(r, z, t), v_{\varphi}(r, z, t), v_{z}(r, z, t)\right)$, we obtain that $C_{2}=0$. Without loss of generality we chose $C_{1}=1$ and the solution takes the form:

$\psi(r, z, t)=\varepsilon\left(z-z^{2}\right) \frac{z}{\alpha(t)(r-1) \sqrt{z^{2}+r^{2}}} \quad$ in $\widehat{\Omega}^{\tau} \cup \hat{\mathrm{S}}_{2}^{\tau} ;$

$\psi(1, z, t)=0 \quad$ on $\quad \hat{\mathrm{S}}_{1}^{\tau}$

this ends the proof.

In order to solve the equation (13) the following statement holds

\section{Proposition 2 .}

The equation (13) with the boundary conditions (9) has the following solution $T(r, z, t)=\frac{t_{0}-t_{1}-\tilde{T}}{h\left(s_{1}\right)} h(s)+\tilde{T}-t_{o}+t, \quad$ in $\widetilde{\Omega}^{\tau} \cup{\widehat{S_{2}}}^{\tau} ;$

$T(1, z, t)=\tilde{T}-t_{o}+t+K\left(t_{o}-t_{1}-\tilde{T}\right) \int_{0}^{\xi} \exp \left(\frac{\alpha^{\prime}}{2 \alpha^{2}} \zeta^{2}\right) d \zeta \quad$ on ${\widehat{\mathrm{S}_{1}}}^{\tau}$

where

$$
\begin{aligned}
& h(s)=\int_{0}^{s} \exp (-\chi(\eta)) d \eta ; \quad K=\left[\int_{0}^{\alpha\left(t_{1}\right)} \exp \left(\frac{\alpha^{\prime}}{2 \alpha^{s}} \zeta^{2}\right) d \zeta\right]^{-1} ; \\
& \chi(s)=-\frac{\alpha^{\prime}}{\alpha} z^{2} \ln |s|+\frac{1}{2}\left(1+\frac{\alpha^{\prime}}{\alpha} z^{2}\right) \ln \left(s^{2}+\alpha^{2}\right)+\varepsilon\left(z-z^{2}\right)\left\{-\frac{s^{2}}{(s-\alpha z)^{2} \sqrt{s^{2}+\alpha^{2}}}\right. \\
& +\left[\frac{z^{2}}{\left(z^{2}+1\right)(s-\alpha z)^{2}}+\frac{z\left(-z^{2}+2\right)}{\alpha\left(z^{2}+1\right)^{2}(s-\alpha z)}\right] \\
& \times \sqrt{(s-\alpha z)^{2}+2 \alpha z(s-\alpha z)+\alpha^{2}\left(z^{2}+1\right)} \\
& -\frac{3 z^{2}}{\alpha\left(z^{2}+1\right)^{\frac{5}{2}}} \ln \mid \frac{1}{s-\alpha z}+\frac{z}{\alpha\left(z^{2}+1\right)} \\
& \left.+\frac{\sqrt{(s-\alpha z)^{2}+2 \alpha z(s-\alpha z)+\alpha^{2}\left(z^{2}+1\right)}}{\alpha(s-\alpha z) \sqrt{z^{2}+1}}\right\} \\
& s=\frac{\alpha z}{r} ; \xi=\alpha z ; \\
& t=t_{0}, t=t_{1} \text { correspond respectively to } z=0, \quad z=1 .
\end{aligned}
$$




\section{Proof}

Putting $T(r, z, t)=h(s)+t, s=\frac{\alpha z}{r}, \forall(r, z, t) \in \widehat{\Omega}^{\tau} \cup{\widehat{S_{2}}}^{\tau}$ the equation (13) leads to $h^{\prime \prime}(s)+g(s) h^{\prime}(s)=0$

which produces the solution satisfying the boundary conditions (9)

$T(r, z, t)=\frac{t_{0}-t_{1}-\tilde{T}}{h\left(s_{1}\right)} h(s)+t+\tilde{T}-t_{0}, \quad \forall(r, z, t) \in \widehat{\Omega}^{\tau} \cup{\widehat{S_{2}}}^{\tau}$

where

$g(s)=\frac{s-\alpha^{\prime} z r-s \frac{\partial \psi}{\partial z}-\alpha \frac{\partial \psi}{\partial r}}{s^{2}+\alpha^{2}}=\frac{s^{2}-\alpha^{\prime} \alpha^{2} z^{2}}{s\left(s^{2}+\alpha^{2}\right)}+\frac{s^{8} s\left(z-z^{2}\right)}{(s-\alpha z)^{2}\left(s^{2}+\alpha^{2}\right)^{\frac{8}{2}}}$

$h(s)=\int_{0}^{s} \exp (-\chi(\eta)) d \eta ; \quad \frac{d \chi}{d s}=g(s) ;$

$\chi(s)=-\frac{\alpha^{\prime}}{\alpha} z^{2} \ln |s|+\frac{1}{2}\left(1+\frac{\alpha^{\prime}}{\alpha} z^{2}\right) \ln \left(s^{2}+\alpha^{2}\right)$

$+\varepsilon\left(z-z^{2}\right)\left\{-\frac{s^{2}}{(s-\alpha z)^{2} \sqrt{s^{2}+\alpha^{2}}}\right.$

$+\left[\frac{z^{2}}{\left(z^{2}+1\right)(s-\alpha z)^{2}}+\frac{z\left(-z^{2}+2\right)}{\alpha\left(z^{2}+1\right)^{2}(s-\alpha z)}\right]$

$\times \sqrt{(s-\alpha z)^{2}+2 \alpha z(s-\alpha z)+\alpha^{2}\left(z^{2}+1\right)}$

$-\frac{3 z^{2}}{\alpha\left(z^{2}+1\right)^{\frac{5}{2}}} \ln \mid \frac{1}{s-\alpha z}+\frac{z}{\alpha\left(z^{2}+1\right)}$

$\left.+\frac{\sqrt{(s-\alpha z)^{2}+2 \alpha z(s-\alpha z)+\alpha^{2}\left(z^{2}+1\right)}}{\alpha(s-\alpha z) \sqrt{z^{2}+1}}\right\}$.

On the set ${\widehat{\mathrm{S}_{1}}}^{\tau}, \psi(1, z, t)=0$ and the equation (13) leads to

$\frac{\partial T(1, z, t)}{\partial t}=\frac{\partial^{2} T(1, z, t)}{\partial z^{2}}+1$

which produces the solution :

$T(1, z, t)=\tilde{T}-t_{0}+t+K\left(t_{0}-t_{1}-\tilde{T}\right)\left(\int_{0}^{\xi} \exp \left(\frac{\alpha^{\prime}}{2 \alpha^{3}} \zeta^{2}\right)\right) d \zeta \quad$ on ${\widehat{\mathrm{S}_{1}}}^{\tau} ; \xi=a z ;$

this ends the proof.

Let us determine the azimuthal component $v_{\varphi}$ satisfying the boundary conditions $v_{\varphi \mid z=0,1}=0$.

Thus the following statement holds

\section{Proposition 3 .}

The solution $v_{\varphi}$ of the equation (11) satisfying the boundary conditions $v_{\varphi}=0$ on $\widehat{\mathrm{S}}_{2}^{\tau}$ has the following form:

$v_{\varphi}^{2}=A+\operatorname{Pr} \times B+C+D$

Where

$$
\begin{aligned}
& A=\frac{\alpha^{\prime} r^{2} \varepsilon\left(z-z^{2}\right)}{\alpha^{2}(r-1)\left(z^{2}+r^{2}\right)^{\frac{8}{2}}}-r \frac{3 z^{4}+\left(5 r^{2}-r\right) z^{2}+2 r^{4}-r^{8}}{\alpha^{2}(r-1)^{3}\left(z^{2}+r^{2}\right)^{4}}+\frac{z^{2}\left(z^{2}+2 r^{2}-r\right)\left[2 z^{4}+\left(5 r^{2}-1\right) z^{2}+6 r^{4}-6 r^{8}+2 r^{2}\right]}{\alpha^{2} r(r-1)^{5}\left(z^{2}+r^{2}\right)^{4}} \\
& +\frac{z^{2}\left(z^{2}+2 r^{2}-r\right)^{2}-2 r^{4}(r-1)^{2}}{2 r^{2} \alpha^{2}(r-1)^{4}\left(z^{2}+r^{2}\right)^{3}} \\
& B=\frac{2 s\left(z-z^{2}\right)\left[10 z^{4}+\left(14 r^{2}+3 r-5\right) z^{2}+8 r^{4}-9 r^{8}+3 r^{2}\right]}{\alpha(r-1)^{2}\left(z^{2}+r^{2}\right)^{\frac{5}{2}}}-\frac{s\left(z-z^{2}\right)}{\alpha(r-1)^{3}\left(z^{2}+r^{2}\right)^{\frac{7}{2}}}\left[20 z^{6}+10\left(5 r^{2}-1\right) z^{4}+\left(48 r^{4}-36 r^{3}+\right.\right. \\
& \left.\left.8 r^{2}\right) z^{2}+3 r^{6}-6 r^{5}+3 r^{4}\right]+R a\left[\frac{\left(\hat{T}+t_{1}-{ }_{0}\right) z h(s)}{h\left(s_{1}\right)}+\frac{t_{0}-t_{1}-\tilde{T}}{h\left(s_{1}\right)} \int_{0}^{z} h(s) d s\right]
\end{aligned}
$$




$$
\begin{aligned}
& -3 r \frac{\varepsilon\left(z-z^{2}\right)}{\alpha(r-1)^{4}\left(z^{2}+r^{2}\right)^{\frac{7}{2}}}\left[\frac{\left(-15 r^{3}+11 r^{2}-5 r+1\right)\left(z^{2}+r^{2}\right)^{4}}{r^{4}(r-1)}\right. \\
& \left.+8\left(z^{2}+r^{2}\right)^{3}+2\left(r^{2}-1\right)\left(z^{2}+r^{2}\right)^{2}+4 r(r-1)^{2}\left(z^{2}+r^{2}\right)+5 r^{3}(r-1)^{3}\right] ; \\
& C=-r \frac{\varepsilon\left(z-z^{2}\right) \alpha^{\prime}}{\alpha^{2}(r-1)^{3}\left(z^{2}+r^{2}\right)^{\frac{8}{2}}}\left[\frac{3 r-1}{r^{2}}\left(z^{2}+r^{2}\right)^{2}+2(r-1)\left(z^{2}+r^{2}\right)\right. \\
& \left.+r(r-1)^{2}\right]+\frac{3}{4 \alpha^{2} r^{2}(r-1)^{3}}\left[\frac{2 z^{2}+3 r^{2}-r}{\left(z^{2}+r^{2}\right)^{2}}-\frac{3}{r^{2}}+\frac{1}{r^{2}}\right] \\
& +\frac{3}{\alpha^{2} r(r-1)^{4}\left(z^{2}+r^{2}\right)^{8}}\left[-\frac{\left(7 r^{2}-4 r+1\right)\left(z^{2}+r^{2}\right)^{3}}{4 r^{4}}\right. \\
& \left.+\left(z^{2}+r^{2}\right)^{2}+\frac{1}{4}\left(r^{2}-1\right)\left(z^{2}+r^{2}\right)+\frac{1}{2} r^{2}(r-1)^{2}\right] \\
& D=\frac{r-2}{\alpha^{2} r(r-1)^{5}\left(z^{2}+r^{2}\right)^{4}}\left[\frac{1}{24 r^{4}}\left(17 r^{2}-6 r+1\right)\left(z^{2}+r^{2}\right)^{4}-\left(z^{2}+r^{2}\right)^{3}\right. \\
& \left.+\frac{1}{4}\left(r^{2}+1\right)\left(z^{2}+r^{2}\right)^{2}-\frac{1}{3} r^{2}\left(r^{2}-3 r+2\right)\left(z^{2}+r^{2}\right)+\frac{3}{8} r^{4}(r-1)^{2}\right] \\
& +\frac{2 r^{2}-r}{\alpha^{2}(r-1)^{5}\left(z^{2}+r^{2}\right)^{4}}\left[-\frac{1}{24 r^{6}}\left(25 r^{2}-18 r+5\right)\left(z^{2}+r^{2}\right)^{4}\right. \\
& \left.+\frac{1}{2}\left(z^{2}+r^{2}\right)^{2}+\frac{1}{6}\left(r^{2}-1\right)\left(z^{2}+r^{2}\right)+\frac{3}{8} r^{2}(r-1)^{2}\right] \\
& \frac{3}{\alpha^{2}(r-1)^{5}\left(z^{2}+r^{2}\right)^{4}}\left[-\frac{1}{8 r^{5}}\left(15 r^{3}-11 r^{2}+5 r-1\right)\left(z^{2}+r^{2}\right)^{4}\right. \\
& \left.+\left(z^{2}+r^{2}\right)^{3}+\frac{1}{4}\left(r^{2}-1\right)\left(z^{2}+r^{2}\right)^{2}+\frac{1}{2} r(r-1)^{2}\left(z^{2}+r^{2}\right)+\frac{5}{8} r^{3}(r-1)^{3}\right]
\end{aligned}
$$

\section{Proof}

Applying the operators $-\frac{\partial}{\partial z}$ and $\frac{\partial}{\partial r}$ to the relations (10), (12) respectively and suming the obtained results, we get

$$
\begin{aligned}
& v_{\varphi}^{2}(r, z, t)=r\left\{\frac{\partial v_{r}}{\partial t}+\frac{1}{2} \frac{\partial v_{r}^{2}}{\partial r}+v_{z} \frac{\partial v_{r}}{\partial z}-\frac{1}{2} \frac{\partial v_{z}^{2}}{\partial r}\right. \\
& -\operatorname{Pr}\left[\frac{\partial\left(\frac{1 \partial r v_{r}}{r r}\right)}{\partial r}+\frac{\partial^{2} v_{r}}{\partial z^{2}}-\frac{\partial^{2} v_{z}}{\partial z \partial r}\right] \\
& +\int_{0}^{z}\left[\operatorname{Pr}\left(\operatorname{Ra} \frac{\partial T}{\partial r}+\frac{\partial}{\partial r}\left(\frac{1}{r} \frac{\partial}{\partial r}\left(\frac{r \partial v_{z}}{\partial r}\right)\right)\right)\right. \\
& \left.\left.-\frac{\partial^{2} v_{z}}{\partial r \partial t}-\frac{\partial\left(v_{r} \frac{\partial v_{z}}{\partial r}\right)}{\partial r}\right] d z\right\}
\end{aligned}
$$

which can be written through the stream function $\psi$ as follows :

$$
\begin{aligned}
& v_{\varphi}^{2}(r, z, t)=\frac{-\partial^{2} \psi}{\partial t \partial z}+\frac{1}{r} \frac{\partial \psi}{\partial z} \frac{\partial^{2} \psi}{\partial z \partial r}-\frac{1}{r} \frac{\partial \psi}{\partial r} \frac{\partial^{2} \psi}{\partial r^{2}}-\frac{1}{r^{2}}\left(\frac{\partial \psi}{\partial z}\right)^{2} \\
& -\frac{1}{r} \frac{\partial \psi}{\partial r} \frac{\partial^{2} \psi}{\partial z^{2}}+\frac{1}{2 r^{2}}\left(\frac{\partial \psi}{\partial r}\right)^{2}+\operatorname{Pr}\left(\frac{-2}{r} \frac{\partial^{2} \psi}{\partial r \partial z}+2 \frac{\partial^{3} \psi}{\partial r^{2} \partial z}+\frac{\partial^{3} \psi}{\partial z^{3}}\right)
\end{aligned}
$$


$+\int_{0}^{z}\left[\operatorname{Pr}\left(\operatorname{Rar} \frac{\partial T}{\partial r}-\frac{3}{r^{3}} \frac{\partial \psi}{\partial r}+\frac{3}{r^{2}} \frac{\partial^{2} \psi}{\partial r^{2}}-\frac{2}{r} \frac{\partial^{3} \psi}{\partial r^{3}}\right.\right.$

$\left.+\frac{\partial^{4} \psi}{\partial r^{4}}\right)+\frac{1}{r} \frac{\partial^{2} \psi}{\partial t \partial r}-\frac{\partial^{3} \psi}{\partial t \partial r^{2}}+\frac{3}{r^{3}} \frac{\partial \psi}{\partial z} \frac{\partial \psi}{\partial r}$

$\left.-\frac{3}{r^{2}} \frac{\partial \psi}{\partial z} \frac{\partial^{2} \psi}{\partial r^{2}}+\frac{1}{r} \frac{\partial^{2} \psi}{\partial r \partial z} \frac{\partial^{2} \psi}{\partial r^{2}}+\frac{1}{r} \frac{\partial \psi}{\partial z} \frac{\partial^{3} \psi}{\partial r^{3}}\right] d z$.

Putting

$A=-\frac{\partial^{2} \psi}{\partial t \partial z}+\frac{1}{r} \frac{\partial \psi}{\partial z} \frac{\partial^{2} \psi}{\partial z \partial r}-\frac{1}{r} \frac{\partial \psi}{\partial r} \frac{\partial^{2} \psi}{\partial r^{2}}$

$-\frac{1}{r^{2}}\left(\frac{\partial \psi}{\partial z}\right)^{2}-\frac{1}{r} \frac{\partial \psi}{\partial r} \frac{\partial^{2} \psi}{\partial z^{2}}+\frac{1}{2 r^{2}}\left(\frac{\partial \psi}{\partial r}\right)^{2}$

$B=-\frac{2}{r} \frac{\partial^{2} \psi}{\partial r \partial z}+2 \frac{\partial^{3} \psi}{\partial r^{2} \partial z}+\frac{\partial^{3} \psi}{\partial z^{3}}+\int_{0}^{z}\left(R a \frac{\partial T}{\partial r}-\frac{3}{r^{3}} \frac{\partial \psi}{\partial r}\right.$

$\left.+\frac{3}{r^{2}} \frac{\partial^{2} \psi}{\partial r^{2}}-\frac{2}{r} \frac{\partial^{3} \psi}{\partial r^{3}}+\frac{\partial^{4} \psi}{\partial r^{4}}\right) d z ;$

$C+D=\int_{0}^{z}\left[\frac{1}{r} \frac{\partial^{2} \psi}{\partial t \partial r}-\frac{\partial^{2} \psi}{\partial t \partial r^{2}}+\frac{3}{r^{3}} \frac{\partial \psi}{\partial z} \frac{\partial \psi}{\partial r}-\frac{3}{r^{2}} \frac{\partial \psi}{\partial z} \frac{\partial^{2} \psi}{\partial r^{2}}\right.$

$\left.+\frac{1}{r} \frac{\partial^{2} \psi}{\partial r \partial z} \frac{\partial^{2} \psi}{\partial r^{2}}+\frac{1}{r} \frac{\partial \psi}{\partial z} \frac{\partial^{s} \psi}{\partial r^{8}}\right] d z$

and computing the derivatives of the functions in $A, B, C, D$ we get the expression of $v_{\varphi}$; this ends the proof. In order to compute the cylindrical components of the vorticity vector, we formulate the following.

\section{Proposition 4.}

Let $\omega=\frac{1}{2}$ rotv be a vorticity vector. Then the cylindrical components of $\omega$ have the following form:

$$
\begin{aligned}
& \omega_{r}=\frac{1}{4 v_{\varphi}}\left(\frac{\partial A}{\partial z}+\operatorname{Pr} \frac{\partial B}{\partial z}+\frac{\partial C}{\partial z}+\frac{\partial D}{\partial z}\right) ; \\
& \omega_{\varphi}=-\frac{z \varepsilon\left(z-z^{2}\right)}{2 a r^{2}(r-1)^{8}\left(z^{2}+r^{2}\right)^{\frac{5}{2}}}\left[(3 r-1) z^{4}+r\left(r^{4}-3 r^{3}+6 r^{2}-1\right) z^{2}+r^{4}(5 r-3)\right] ; \\
& \omega_{z}=\frac{v_{\varphi}}{2 r}+\frac{1}{4 v_{\varphi}}\left(\frac{\partial A}{\partial r}+\operatorname{Pr} \frac{\partial B}{\partial r}+\frac{\partial C}{\partial r}+\frac{\partial D}{\partial r}\right), \quad \forall(r, z, t) \in \widetilde{\Omega}^{\tau} \cup \widehat{S}_{2}^{\tau} .
\end{aligned}
$$

\section{Proof}

Using the explicit relations between the cylindrical components of the vorticity and velocity, we get:

$$
\begin{aligned}
& 2 \omega_{r}=-\frac{1}{r} \frac{\partial\left(r v_{\varphi}\right)}{\partial z} ; \\
& 2 \omega_{\varphi}=\frac{\partial v_{r}}{\partial z}-\frac{\partial v_{z}}{\partial r} ; \\
& 2 \omega_{z}=\frac{1}{r} \frac{\partial\left(r v_{\varphi}\right)}{\partial r} ;
\end{aligned}
$$

this ends the proof. 


\section{Conclusion}

In this paper, we have investigated the exact solutions to the Navier Stokes equations in a cylinder containing a viscous incompressible fluid. Furthermore, we have investigated the components of the vorticity vector which characterize the turbulence of the fluid and have determined the temperature field of the fluid.

\section{References}

[1] Lu Lu; Doering C.R.; Busse F.H. Bounds on convection driven by internal heating. J. Math. Phys., Vol. 45, No. 7, 2968-2985; July 2004.

[2] Bobok Elemer; Navratil Laszlo; Muszaki Fizika 1: Aramlastan.Kezirat, Tankonyvkiado, Budapest 1990.

[3] Eglit M. E. and al. Problemes de mecanique des milieux continus. Tomes 1, 2. Lycee Moscovite, 1996.

[4] Busse, F. On Howard's upper bound for heat transport by turbulent convection. J. Fluid Mech. 37, 457-477 1969.

[5] Busse, F. The bounding theory of turbulence and its physical significance in the case of turbulent Couette flow. In: Statistical Models and Turbulence, edited by M. Rosenlatt and C. M. Van Atta, Springer Lecture Notes in Physics Vol. 12 (Springer, Berlin, 1972), pp. 103 -126.

[6] Busse, F. The optimum theory of turbulence. Adv. Appl. Mech. 18, 77 -121, 1978.

[7] Busse, F. On the optimum theory of turbulence, Energy Stability and convection, Pitman Research Notes in Mathematics, edited by G. Galdi and B. Straughan, Wiley, New York, 1987.

[8] Zadrzynska E.; Zajaczkowski W.M. Global Regular Solutions with Large Swirl to the NavierStokes Equations in a cylinder. J. Math. Fluid Mechanics; Vol. 11, 126-169, 2009. 